\title{
Plant genetic resources in India: management and utilization
}

Singh K.*, Gupta K., Tyagi V., Kumar R.S.

ICAR - National Bureau of Plant Genetic Resources, New Delhi, India

*e-mail: Kuldeep.Singh4@icar.gov.in

Plant genetic resources (PGR) are the foundation of agriculture as well as food and nutritional security. The ICAR-NBPGR is the nodal institution at national level for management of PGR in India under the umbrella of Indian Council of Agricultural Research (ICAR), New Delhi. India being one of the gene-rich countries faces a unique challenge of protecting its natural heritage while evolving mutually beneficial strategies for germplasm exchange with other countries. The Bureaus activities include PGR exploration, collection, exchange, characterization, evaluation, conservation and documentation. It also has the responsibility to carry out quarantine of all imported PGR including transgenics meant for research purposes. The multifarious activities are carried out from ICAR-NBPGR headquarters and its 10 regional stations located in different agro-climatic zones of India. It has linkages with international organizations of the Consultative Group on International Agricultural Research (CGIAR) and national crop-based institutes to accomplish its mandated activities. NBPGR collects and acquires germplasm from various sources, conserves it in the Genebank, characterizes and evaluates it for different traits and provides ready material for breeders to develop varieties for farmers. ICAR-NBPGR encompasses the National Genebank Network and at present, the National Genebank conserves more than 0.40 million accessions. NBPGR works in service-mode for effective utilization of PGR in crop improvement programmes which depends mainly on its systematic characterization and evaluation, and identification of potentially useful germplasm. NBPGR is responsible for identifying trait-specific pre-adapted climate resilient genotypes, promising material with disease resistance and quality traits which the breeders use for various crop improvement programmes. The system has contributed immensely towards safeguarding the indigenous and introducing useful exotic PGR for enhancing the agricultural production. Presently, our focus is on characterization of ex situ conserved germplasm and detailed evaluation of prioritized crops for enhanced utilization; assessment of impact of on-farm conservation practices on genetic diversity; genome-wide association mapping for identification of novel genes and alleles for enhanced utilization of PGR; identification and deployment of germplasm/landraces using climate analog data; validation of trait-specific introduced germplasm for enhanced utilization. Gene banks have often, through necessity, focused mainly on the immediate but long-term conservation of plant genetic resource activities. There is currently a major gulf between the operations of PGR collections and modern plant breeding. The conservation of genetic resources must be linked to their increased and sustainable use if they are to play a key role in climate change adaptation. This could be achieved through active engagement with all stakeholders in order to assure the functionality of the entire "Genetic resource-chain". The recent progress in genomics has opened up enormous possibilities, both for introgression of specific traits and for base broadening in pre-breeding. Development of molecular markers since 1980s has seen a striding development from RFLPs to SNPs because of progress in high throughput genomics at very low costs. A few case studies on alien introgression in rice, wheat, lentil, and Brassica involving use of genomics tools for rapid introgression of desirable variability from un-adapted germplasm to high yielding varieties in India and their commercialization will be presented. 